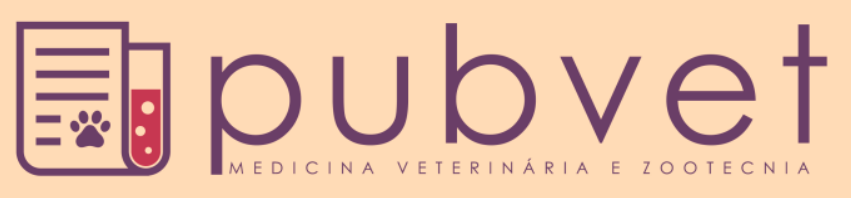

https://doi.org/10.31533/pubvet.v12n10a197.1-5

\title{
Osteossarcoma renal em cadela: Relato de caso
}

\author{
Bruna Roberta Milagres Araújo ${ }^{1 *} \bullet$, Valesca Oliveira de Sousa $^{2} \bullet$, Ana Paula Aragão ${ }^{30}$ \\ Mariana Gonçalves de Andrade Paiva ${ }^{4} \bullet$
}

${ }^{I}$ Graduanda da Faculdade de Medicina Veterinária de Valença-Valença - RJ Brasil.

${ }^{2}$ Professora da Faculdade de Medicina Veterinária de Valença-Departamento de Patologia - Valença-RJ Brasil.

${ }^{3}$ Professora da Faculdade de Medicina Veterinária de Valença-Departamento de Patologia - Valença-RJ Brasil.

${ }^{4}$ Médica Veterinária - Valença-RJ Brasil.

*Autor para correspondência, E-mail: brunamilagres@hotmail.com

RESUMO. Os osteossarcomas ósseos são muito comuns em pequenos animais. Todavia, os osteossarcomas extra ósseos renais primários não; logo os relatos na literatura são raros. O objetivo deste trabalho foi relatar o caso de uma cadela sem raça definida, de meia idade atendida em uma clínica no estado do Rio de Janeiro acometida por osteossarcoma renal. Os sintomas clínicos característicos ao osteossarcoma renal são semelhantes às patologias renais em geral e não apresentam especificidade. Por este motivo devem-se fazer exames complementares como ultrassonografia, radiografia abdominal e exames laboratoriais, por exemplo, para auxiliar no diagnóstico. O animal do caso relatado foi submetido a uma nefrectomia unilateral e para fins de diagnóstico foi realizado exame histopatológico no laboratório Vet. Análises - Diagnóstico Veterinário. Pouco se sabe sobre o prognóstico dessa patologia, pois existem poucos relatos na literatura sobre a evolução desses casos, ficando assim difícil se ter um parâmetro sobre a sobrevida dos animais acometidos. Portanto, pode-se concluir a necessidade de maiores estudos sobre o caso em questão.

Palavras chave: cão, osteossarcoma extra-ósseo, rim

\section{Renal osteosarcoma in female dog: Case report}

ABSTRACT. Bone osteosarcomas are very common to find in small animals, but the osteosarcoma extra-osseous primary renal are not, because of that, relates on literature about it are rare. The propose of this college work was relate the case of a undefined female and middle-aged dog attended on a Rio de Janeiro state's clinic who had a unexpected appearance of a renal osteosarcoma. The characteristic clinical symptoms of renal osteosarcoma are similar to the renal pathologies in general and doesn't show specificity, for this reason, should be make complementary exams like ultrasonography, abdominal radiography and laboratorial exams for example, to help the diagnostic. The animal in case was submitted to a unilateral nephrectomy and for the purpose to diagnostic was made a histopathological exam on Veterinary laboratory tests - Veterinary Diagnosis. Little is known about the prognosis of this pathology, because there too many few relates on literature about the evolution of this kind of case, becoming hard to get a parameter about animal's afterlife with an unexpected disease. Therefore, it's possible to conclude the necessity of more searches about this case.

Key words: Extra-osseous osteosarcoma, Kidney, Dog.

\section{Osteosarcoma renal en perra: Relato de caso}

RESUMEN. Los osteosarcomas óseos son muy comunes de ser encontrados en pequeños animales, pero los osteosarcomas extraóseos renales primarios no, luego los relatos en la 
literatura son raros. El objetivo de este trabajo fue relatar el caso de una perra criolla, de mediana edad atendida en una clínica en el estado de Río de Janeiro afectada por osteosarcoma renal. Los síntomas clínicos característicos del osteosarcoma renal son similares a las patologías renales en general y no presentan especificidad, por lo que se deben realizar exámenes complementarios como ultrasonografía, radiografía abdominal y exámenes de laboratorio, por ejemplo, para auxiliar en el diagnóstico. El animal del caso relatado fue sometido a una nefrectomía unilateral, y para fines de diagnóstico se realizó un examen histopatológico en el laboratorio Vet. Análisis - Diagnóstico Veterinario. Poco se sabe sobre el pronóstico de esa patología, pues hay pocos relatos en la literatura sobre la evolución de esos casos, quedando así difícil tener un parámetro sobre la supervivencia de los animales acometidos. Por lo tanto, se puede concluir la necesidad de mayores estudios sobre el caso en cuestión.

Palabras clave: Osteosarcoma extraóseo, riñón, perro

\section{Introdução}

O rim é responsável por funções reguladoras, excretoras e endócrinas, sendo essencial para a manutenção do equilíbrio hídrico, eletrolítico, homeostático e acidobásico (Strasinger, 1996). O comprometimento do parênquima renal por alguma patologia, entre elas tumores malignos, neoplasias, infestações parasitárias, entre outras citadas na literatura, acarretará em problemas sistêmicos ao organismo humano ou animal.

Os osteossarcomas, dependendo de sua localização, podem ser divididos em esquelético e extra-esqueléticos. Os osteossarcomas de partes moles extra-esqueléticas são associado a um prognóstico extremamente pobre e com um tempo médio de sobrevivência de 26 dias (Langenbach et al., 1998). Entretanto, Weingärtner et al. (1995) citaram tempo de sobrevida de 18 meses após o diagnóstico em seres humanos, sendo o mesmo baseado em exames radiográficos e histológicos. Cirurgia, quimioterapia e radioterapia são muitas vezes tratamentos paliativos para este tumor. Os osteossarcomas renais são considerados tumores malignos e raros em cães (Pool, 1990).

O presente trabalho teve como objetivo relatar um caso de osteossarcoma renal num cão no estado do Rio de Janeiro.

\section{Relato de caso}

Uma cadela, sem raça definida, com doze anos de idade, não castrada, apresentando aumento de volume em região abdominal, dor a palpação, febre, apatia e perda de apetite foi encaminhada por uma ONG para uma clínica particular, localizada na cidade do Rio de Janeiro, para realizar uma castração com suspeita de piometra.

Na clínica constatou-se que o animal não apresentava piometra, com isso foi encaminhada para um procedimento cirúrgico de laparotomia exploratória para pesquisar a enfermidade que poderia estar cursando com os sinais clínicos apresentados. Não foi realizado nenhum exame laboratorial, pois o proprietário do animal não possuía recursos financeiros para arcar com os custos dos exames pré-operatórios. $\mathrm{O}$ animal foi encaminhado, após avaliação clínica, para sala de preparação anestésica, onde recebeu como medicação pré-anestésica: $0,05 \mathrm{mg} \mathrm{kg}$ de maleato de acepromazina $1 \%$, associado a $0,5 \mathrm{mg} / \mathrm{kg}$ de morfina, ambos pela via intramuscular. Uma vez feita a tricotomia da área cirúrgica abdominal, o acesso venoso cefálico foi cateterizado para fluidoterapia na taxa de $5 \mathrm{~mL} / \mathrm{kgh}$. O animal foi transferido para o centro cirúrgico onde recebeu a indução anestésica com propofol $10 \mathrm{mg}$, na dose de $5 \mathrm{mg} \mathrm{kg}$, pela via intravenosa, e manutenção anestésica com 1,5 CAN de isoflurano pela via inalatória. Um bloqueio epidural com cloridrato de lidocaína a $2 \%$ na dose de $5 \mathrm{mg} / \mathrm{kg}$ e associado a morfina $0,1 \mathrm{mg} / \mathrm{kg}$ sem vasoconstrictor foi realizado para o controle da dor pela injeção do anestésico no espaço peridural, que se localiza entre as paredes do canal vertebral e a dura máter.

Durante a laparotomia exploratória foi observada uma formação nodular medindo aproximadamente 15 centímetros em topografia de rim esquerdo. Os demais órgãos não apresentaram alterações macroscópicas. Durante o procedimento optou-se pela nefrectomia unilateral do rim afetado (Figura 1).

Após a remoção cirúrgica do rim esquerdo foi observado que o mesmo apresentava perda de sua conformação anatômica com a presença de superfície externa multinodular, de tonalidade ora creme, ora brancacenta ou pardacenta (Figura 2) e, ao corte, observou-se consistência firme-rangente, multilobada com pequena quantidade de conteúdo castanho (Figura 3). A peça cirúrgica foi fixada em formol a $10 \%$ e encaminhada para exame histopatológico. 
Após a recuperação anestésica o animal recebeu alta cirúrgica sendo liberado para casa com prescrição de meloxicam $0,5 \mathrm{mg}$, na dose de $0,1 \mathrm{mg} / \mathrm{kg}$ e enrofloxacina $50 \mathrm{mg}$, na dose de 5 $\mathrm{mgkg}$. Passados 10 dias de pós-operatório o animal não retornou a clínica na data prevista para retirada dos pontos e nova avaliação clínica. Portanto, não foi possível acompanhar o processo de recuperação animal.

O estudo histopatológico dos fragmentos coletados do parênquima renal revelou intensa infiltração maligna de células pleomórficas em região de córtex renal, com destruição do parênquima restando apenas alguns corpúsculos renais remanescentes. Esse infiltrado era composto por células fusiformes, por vezes globulosas e, no permeio destas células, áreas de depósito extracelular de osteóide. Observou-se ainda áreas de mineralização e diferenciação cartilaginosa (Figuras 4 a 7). Os achados

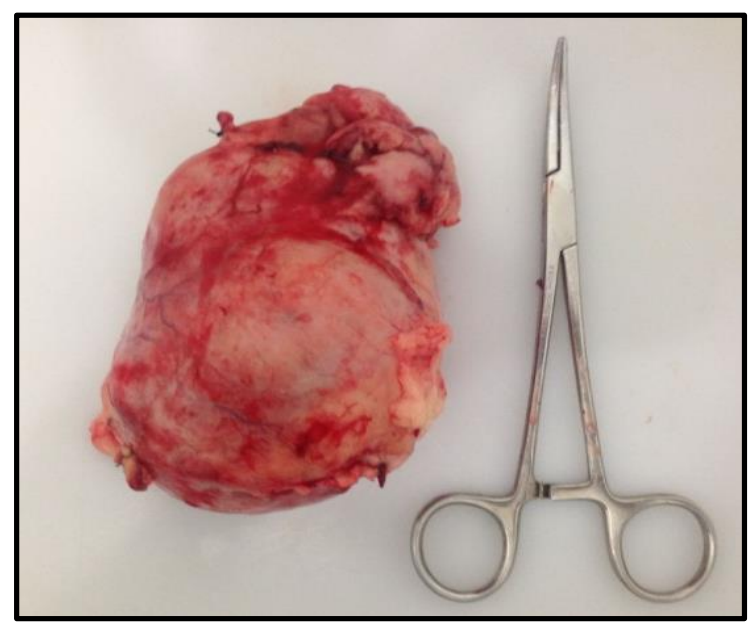

Figura 2. Rim esquerdo com perda de sua conformação anatômica com a presença de superfície externa multinodular.

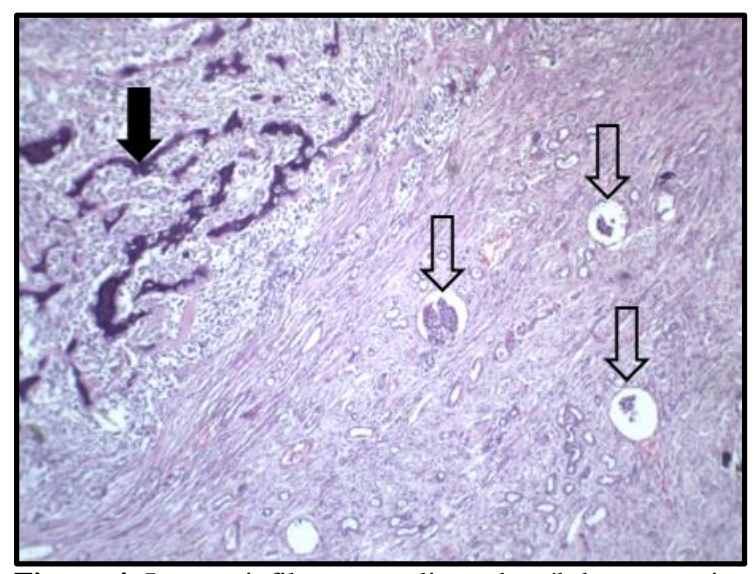

Figura 4. Intensa infiltração maligna de células em região de córtex renal. Observam-se áreas de mineralização (seta cheia) e corpúsculos renais remanescentes (seta). H.E. Obj. $4 \mathrm{x}$. histopatológicos são compatíveis com Osteossarcoma Renal.

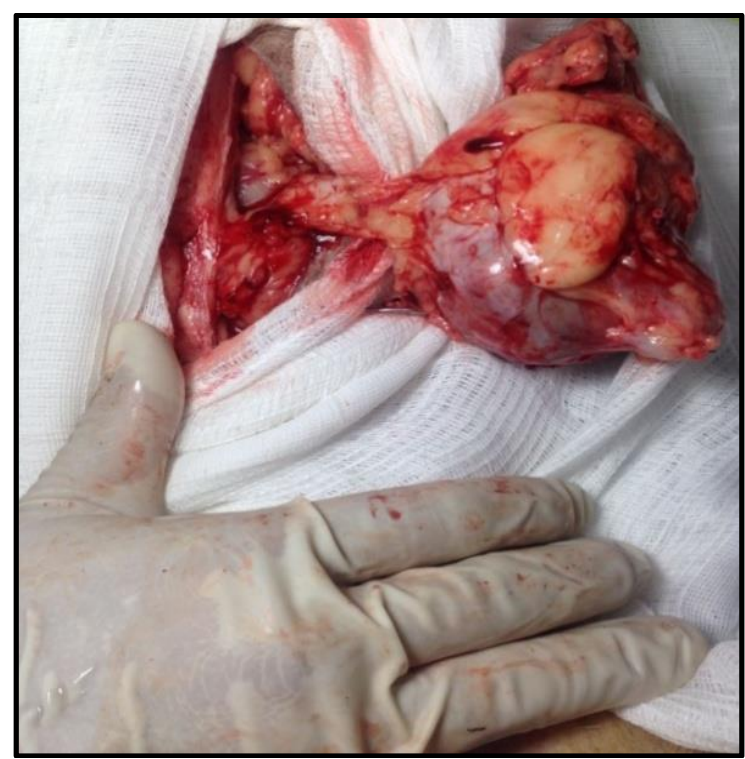

Figura 1. Nefrectomia do rim esquerdo.

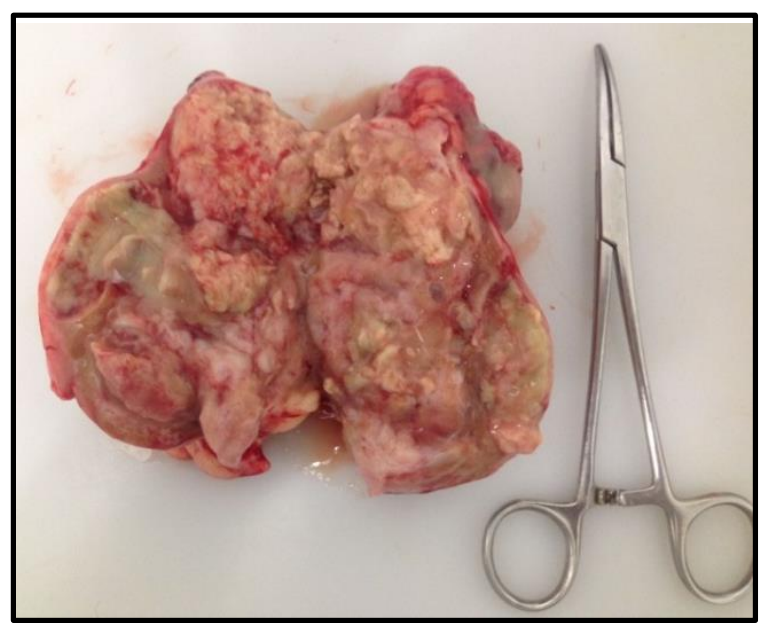

Figura 3. Ao corte a superfície do rim apresentava-se de consistência firme-rangente, multilobada com pequena quantidade de conteúdo castanho.

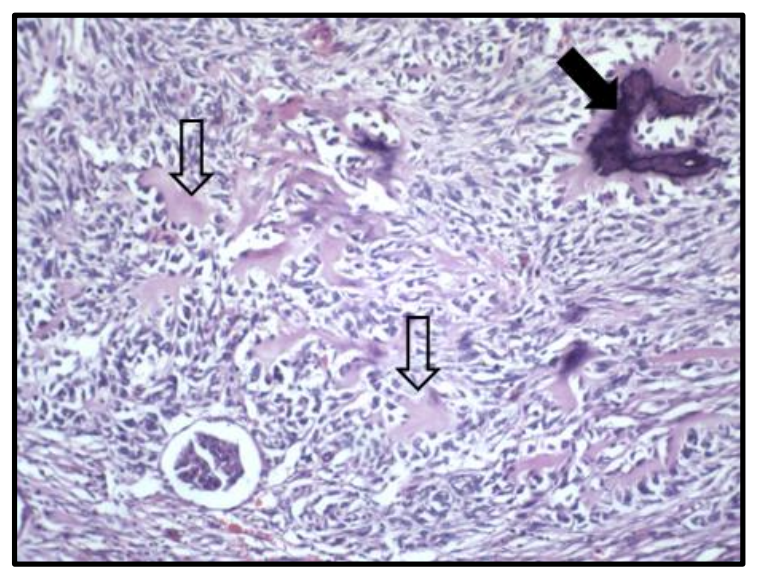

Figura 5. Intensa infiltração maligna de células pleomórficas em região de córtex renal. Observam-se áreas de osteóide (seta), áreas de mineralização (seta cheia) e corpúsculos renais remanescentes. H.E. Obj.10x. 


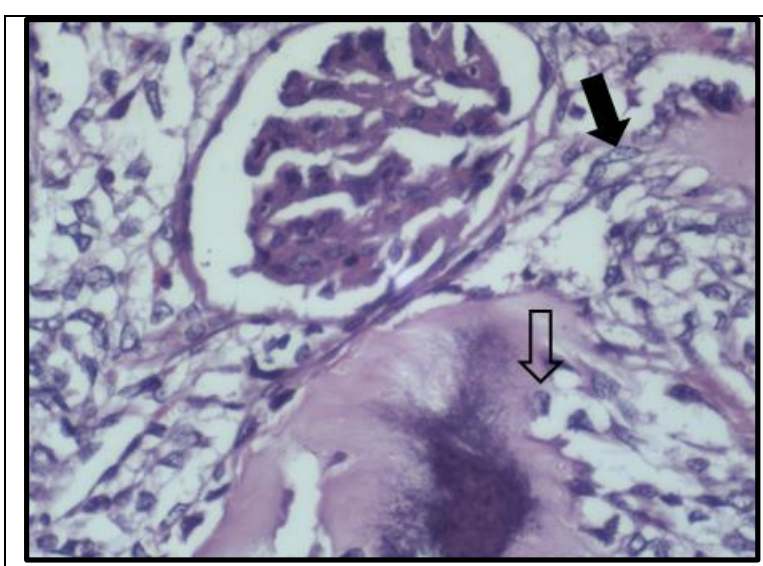

Figura 6. Intensa infiltração maligna de células pleomórficas, fusiformes (seta cheia), por vezes globulosas (seta), com corpúsculo renal remanescente e áreas de osteóide e áreas de mineralização. H.E. Obj.40x

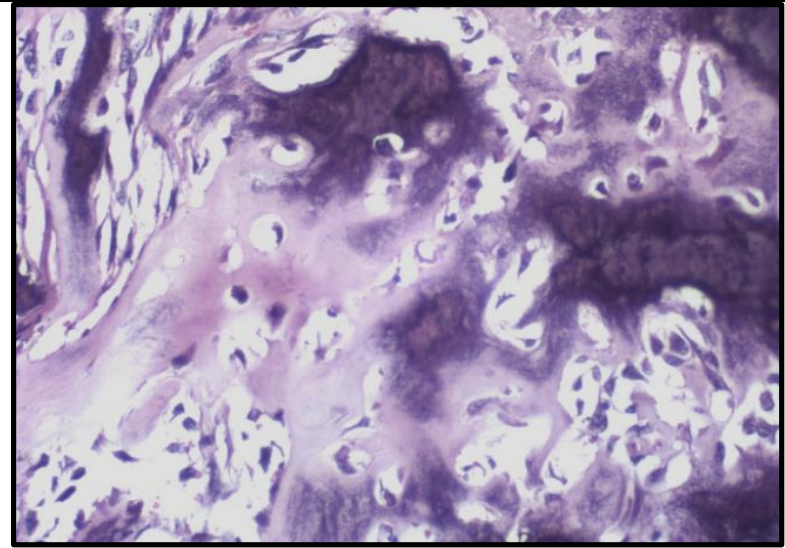

Figura 7. Intensa infiltração maligna de células pleomórficas com destruição do parênquima e áreas de depósito de osteóide e áreas de mineralização. H.E. Obj.40x.

\section{Discussão}

Uma cadela, sem raça definida, com doze anos de idade, não castrada, apresentou uma sintomatologia com aumento de volume em região abdominal, dor a palpação, febre, apatia, e perda de apetite, sinais clínicos compatíveis de acordo como descrito por Puri et al. (2012), os quais constituem-se de uma massa palpavel na região do flanco, dor lombar a palpação, perda de peso e, raramente, hematúria grosseira. Tais sinais que justificam as suspeitas do tumor renal na paciente, a primeiro momento, sem comprovação com exames de imagem como ultrassonografia abdominal.

O osteossarcoma pode ocorrer em qualquer lugar no corpo segundo Kleihues and Cavenee (2000). Thompson and Fugent (1992) citam uma variedade de tecidos que podem ocorrer, incluindo o trato gastrointestinal, tecido subcutâneo, baço, trato urinário, pele, músculo, olho, glândula tireoidiana, glândula salivar, fígado, retroperitônio, pulmão, glândula adrenal, testículo e vagina.

Cirurgia, quimioterapia e radioterapia são muitas vezes tratamentos paliativos para este tumor de acordo com Weingärtner et al. (1995), já Langenbach et al. (1998) relataram que sem terapia complementar, o tempo médio de sobrevivência dos cães é de 26 dias. No entanto, em outro estudo realizado por Kuntz et al. (1998), cães com osteossarcomas de tecidos moles tratados com quimioterapia utilizando cisplatina ou doxorrubicina tem seu tempo de sobrevivência estendido de 33 dias para 146 dias.
Pela laparotomia exploratória o tratamento instituído para o animal em questão foi a realização de excisão tumoral por nefrectomia unilateral esquerda. $\mathrm{E}$ após o procedimento cirúrgico o material (rim esquerdo) foi encaminhado para o exame histopatológico onde Weingärtner et al. (1995) citam que este é o meio correto para se obter o diagnóstico, tendo este como resultado de osteosarcoma em rim.

Sua fisiopatologia, segundo Deshmukh et al. (2012) permanece obscura, na verdade, alguns autores sugerem uma relação entre $o$ osteossarcoma e carcinosarcoma onde o componente mesenquimal supera o componente epitelial e praticamente o faz desaparecer. Já a atual classificação da Organização Mundial da Saúde (OMS) de tumores urogenitais define o osteossarcoma como um processo proliferativo em que as células neoplásicas produzem osteóides no estroma. Já de acordo com Virchow (1884) essas células teriam a capacidade de se diferenciar em células fibroblásticas, condroblásticas e osteoblásticas de acordo com sua clássica teoria, em relação à metaplástica transformação do tecido conjuntivo em mesênquima primitivo com a capacidade de se diferenciar em osteoblastos.

Neste estudo não foram feitos exames referentes à descoberta de metástase, Dimopoulou et al. (2008) descreveram que nos cães, a incidência de metástase por osteossarcoma é de 80 a $90 \%$, e nos gatos esse valor é de 5 a $10 \%$, sendo esse relato considerado incomum. Turrel and Pool (1982) citam que as metástases podem ocorrer em baço, fígado, rins, pulmões e cérebro. Pouco se sabe sobre o prognóstico dessa patologia, pois 
existem poucos relatos na literatura sobre a evolução desses casos, ficando assim difícil se ter um parâmetro. Mas de acordo com Langenbach et al. (1998) osteossarcomas extra esqueléticos de pele e tecido subcutâneo possuem um prognóstico mais favorável do que osteossarcomas que se desenvolvem dentro de órgãos viscerais.

\section{Conclusão}

Em conclusão, a necessidade de que maiores estudos sobre o caso em questão sejam realizados, que uma boa abordagem diagnóstica também é fundamental para o sucesso do tratamento e do prognóstico, o exame ultrassonográfico é uma importante ferramenta complementar para a avaliação renal de cães e de extrema importância para auxiliar no diagnóstico, principalmente no que se refere à identificação do rim acometido e para avaliação do rim contralateral, bem como para avaliar a integridade dos demais órgãos abdominais, fornecendo valiosas informações para o tratamento cirúrgico.

\section{Referências}

Deshmukh, S. D., Gaopande, V. L., Pande, D. P., Pathak, G. S., \& Kulkarni, B. K. 2012. Carcinosarcoma of renal pelvis with immunohistochemical correlation. The Gulf Journal of Oncology, 12, 65-69.

Dimopoulou, M., Kirpensteijn, J., Moens, H., \& Kik, M. 2008. Histologic prognosticators in feline osteosarcoma: a comparison with phenotypically similar canine osteosarcoma. Veterinary Surgery, 37(5), 466-471.

Kleihues, P., \& Cavenee, W. 2000. World Health Organization classification of tumours. Cancer, 88(2887), 1097-0142.

Kuntz, C. A., Dernell, W. S., Powers, B. E., \& Withrow, S. 1998. Extraskeletal osteosarcomas in dogs: 14 cases. Journal of the American Animal Hospital Association, 34(1), 26-30.

Langenbach, A., Anderson, M. A., Dambach, D. M., Sorenmo, K. U., \& Shofer, F. D. 1998. Extraskeletal osteosarcomas in dogs: a retrospective study of 169 cases (1986-1996). Journal of the American Animal Hospital Association, 34(2), 113-120.
Pool, R. 1990. Tumours of bone and cartilage. In J. E. Moulton (Ed.), Tumours of Domestic Animals (pp. 89-149 p.). Berkeley: University of California Press.

Puri, T., Goyal, S., Gupta, R., Julka, P. K., \& Rath, G. K. 2012. Primary renal osteosarcoma with systemic dissemination. Saudi Journal of Kidney Diseases and Transplantation, 23(1), 114-116.

Strasinger, S. K. 1996. Uroanálise e fuídos biológicos. São Paulo, Brasil: Editora Premier.

Thompson, J. P., \& Fugent, M. J. 1992. Evaluation of survival times after limb amputation, with and without subsequent administration of cisplatin, for treatment of appendicular osteosarcoma in dogs: 30 cases (1979-1990). Journal of the American Veterinary Medical Association, 200(4), 531-533.

Turrel, J. M., \& Pool, R. R. 1982. Primary bone tumors in the cat: a retrospective study of 15 cats and a literature review. Veterinary Radiology, 23(4), 152-166.

Virchow, R. 1884. Ueber Metaplasie. Archiv für pathologische Anatomie und Physiologie und für klinische Medicin, 97(3), 410-430.

Weingärtner, K., Gerharz, E. W., Neumann, K., Pflüger, K. H., Grüber, M., \& Riedmiller, H. (1995). Primary osteosarcoma of the kidney. European Urology, 28, 81-84.

Recebido: 28 junho, 2018 Aprovado: 1 agosto, 2018

Publicado: 24 outubro, 2018

Licenciamento: Este artigo é publicado na modalidade Acesso Aberto sob a licença Creative Commons Atribuição 4.0 (CC-BY 4.0), a qual permite uso irrestrito, distribuição, reprodução em qualquer meio, desde que o autor e a fonte sejam devidamente creditados. 